\title{
A Multi-Resolution Accountable Logging and Its Applications
}

\author{
Bo $\mathbf{F u}^{\mathrm{a}}$ and Yang $\mathbf{X i a o}^{\mathrm{b}} *$ \\ ${ }^{a}$ Cisco Systems, Inc., Mail Stop SJC24/2, 510 McCarthy Blvd, Milpitas, CA 95035 USA; \\ ${ }^{\mathrm{b}}$ Department of Computer Science, The University of Alabama, 342 H.M. Comer, Box 870290, Tuscaloosa, AL 35487 USA \\ *Yang Xiao is the corresponding author, Tel: 1-205-348-4038; Fax: 1-205-348-6959 \\ E-mail Addresses: bfu1@ua.edu (B. Fu), yangxiao@ieee.org (Y. Xiao).
}

\begin{abstract}
Today's computer and network systems were not originally designed for accountability which plays a crucial role in information assurance systems. To assure accountability, each entity in the system should be held responsible for its own behaviors so that the entity is part of larger chains of the system's accountability. To achieve accountable logging, a flow-net methodology was proposed in our previous work. Moreover, the multi-layer feature of computer and network systems brings us the chance to achieve multiple degrees of accountability, which means that we are able to acknowledge the system's behaviors at different levels of accountability. To achieve multiple degrees of accountability, we propose designs of a multi-resolution flow-net scheme in this paper and find out the optimal design under different assumptions. Furthermore, we apply the multiresolution flow-net on TCP/IP networks that are designed and organized with multiple layers. Finally, evaluation results are presented to verify the better performance provided by multiresolution flow-net than other schemes.
\end{abstract}

Keywords-Flow-net, accountability, multi-resolution, Computer Networks

\section{Intruduction}

Accountability implies that any entity in a computer or network system should be held responsible for its own specific actions and thus the accountability of the entire system is assured [1]. Ideally, accountability requires that each event that took place in a system should be traceable, and therefore the causes of any action of the system can be determined afterwards. The ability of tracing failures/risks and the responsible entities that caused the failures/risks is a key requirement of accountability.

However, current computer and network systems were not originally designed for accountability, which plays an essential role in information assurance systems [1]. Without assuring accountability, certain sensitive data could be disclosed to unauthorized parties and there might be no effective solution to find out the source and reason of the disclosure [2]. The sensitive data may include confidential records revealed, copyrighted material distributed without permission, and corporate secrets revealed [2].

Therefore, there are some works about the importance and design schemes of accountability proposed. We review the related work in the next section. Particularly, in our previous work, a novel flow-net methodology that builds comprehensive logs and helps track events for accountability was proposed [1].

In this paper, we give a formal definition of flow-net, based on which in a computer system all actions along with their causes are precisely logged. Therefore, flow-net ensures that any entity is held responsible for its actions and thus the accountability of the entire system is assured. Our previous work [1], [10], and [11] did not propose such formal definitions. Moreover, we propose a multi-resolution flow-net logging for multi-layer systems. It is common for a computer system to be organized in a multi-layer manner (e.g., the five-layer TCP/IP networks, internal/secondary memory space, compiler systems). Therefore, making use of the multi-layer structure of a computer and network system is essential for the accountable logging purpose of flow-net. The multi-layer system brings us the chance to achieve multiple degrees of accountability, and this indicates that we are able to acknowledge a system's actions at different layers. Checking the lower layer events that correspond to an event at the higher layer can be considered to be the "zoom in" effect to the event just like the "zoom in" to a picture for higher resolution. For example, as an analogy, we can zoom in the image with a higher resolution in order to see the particular area that we are interested in for more details, and we can also zoom out of the image with a lower resolution in order to get a comprehensive view of the image. Therefore, a multi-resolution flow-net is a flow-net on multi-layers with the zoom feature that is able to show events with a different resolution.

The multi-layer hierarchy structure is used in complicated systems, but it hides the relations between the events in different modules/layers/hierarchies. Events may be related with each other across the modules/layers/hierarchies, because these modules/layers/hierarchies are not isolated; thus, the multi-resolution flow-net is able to record the correlation of the events. The multi-resolution flow-net adapts on the 
complicated hierarchy/multi-layer computer systems and extends the usage of flow-net to achieve multiple degrees of accountability. The multi-resolution feature of flow-net solves the weakness of the non-relation logging scheme, which does not maintain the log as a multi-layer structure as the computer systems are.

The multi-resolution feature of flow-net implies that an event in the higher layer may cause multiple events in the lower layer, and these events are a part of the flow-net in the lower layer. This part of the flow-net in the lower layer is the scope of the higher layer event. The scope of an event is the portion of the flow-net in the lower layer caused by this event. Therefore, we may consider a scope as a set of event's child events. The multi-resolution feature of flow-net provides us with a methodology to trace the relations of events at different layers. In this paper, we propose designs to support distinguishing the scopes of different events in a high layer.

We also discuss on how to deploy multi-resolution flow-net in the actual TCP/IP networks. It is a novel approach to study the accountability of TCP/IP networks by using multiresolution flow-net.

The objective of this paper is to propose a flow-net-based logging scheme aiming at accountability and to apply such a scheme on TCP/IP networks. Although the concept of flow-net is feasible for many types of systems/networks, in this paper we only study multi-resolution flow-net in computer systems and computer networks.

Comparing to our previous work [1], [10], and [11], the contributions that are first made in this work are stated as follows:

- We propose a formal definition of flow-net, instead of the general description in our previous work. The event, flow, scope, and other concepts in flow-net are defined and represented uniquely in a mathematical way. The entire work in this paper is based on these formal definitions.

- We propose multiple designs of multi-resolution flownet schemes. Each of these designs is in accordance with the principle of multi-resolution flow-net.

- The performance of the above designs are defined and evaluated. Optimal designs under different assumptions are built.

- An analysis on how to deploy multi-resolution flow-net in the actual TCP/IP networks is presented. The events of some well-known TCP/IP protocols are studied and listed individually. The layer abstraction of TCP/IP protocols make multi-resolution flow-net naturally adapt with TCP/IP networks.

The rest of this paper is organized as follows. The related work is introduced in Section 2. In Section 3, we present the mathematical definition of flow-net. Different designs of multiresolution flow-net are presented in Section 4. In order to discuss on deploying flow-net in actual TCP/IP networks, the events in TCP/IP networks from the perspective of flow-net are summarized in Section 5. Moreover, a discussion on deploying multi-resolution flow-net in the actual networks is presented in
Section 6. Evaluation results are given in Section 7. We conclude our work in Section 8.

\section{Related Work}

The necessities of introducing accountability in today's computer and network systems are presented in [2-4]. The authors of [5] propose a model to give a formal description of accountability that reveals the "degree of responsibilities" between two events, and the model can be used for formal definition of accountability. However, the work in [5] does not include a specific design scheme for accountability.

Accountability has been considered as the first-class design principle by researchers $[22,25,26,36,49,50,51]$, since trustworthy is a long-term goal for computer systems [27]. In [23], accountability for cloud computing was studied from both consumer and provider side. A logging framework for accountability in distributed systems was proposed in [24]. Accountability designs were proposed for network storage services [28], ISP interface [29], TCP congestion support [30, $31]$, and Future Internet $[32,36]$. Accountability is considered as a key requirement for Electronic commerce. Accountability properties in online life were studied in [37]. An accountability model and protocol for Internet money transaction was proposed in [38]. Principles of accountability were studied from perspectives of E-Health and healthcare in [39]. Due to the aforementioned applications and implementations of accountability, privacy has become a significant concern. Some accountability mechanisms that seek to encourage policycompliant behavior were developed in [40]. The balance between privacy and accountability was discussed in [41]. Accountability in operating system was proposed and studied in $[42,43]$. Accountability for cloud computing was proposed and studied in [44, 45]. Accountability for smart grids was proposed and studied in [46, 47]. Temporal accountability was studied in [48].

Logging is widely used in accountability designs. In the logging schemes that completely record the system and user events, the events leave their trace in the log and thus the log can be used for accountability. There are many kinds of logging schemes, including:

- The transaction log that is widely implemented in the database systems [6].

- A syslog service that is a de facto standard for management and security logging [7].

- A logging scheme for distributed systems that is used for recoverable distributed shared memory systems [8].

- A logging scheme that is used for mobile computing systems [9].

However, current logging schemes treat and record each event individually without considering the relations between the events, and we call them non-relation logging. Without recording the relations between the events, a comprehensive logging that helps effectively track events cannot be built, and thus, the system administrator has to read and analyze the events manually in order to track a certain series of events.

In order to record both events and their relations, a novel flow-net methodology that builds comprehensive logs and helps track events for accountability was proposed in our previous work [1]. In our previous work [10, 11], a preliminary 
implementation of flow-net was presented. Other than nonrelation logging schemes that record only the events in the system, the flow-net scheme records the events and their relations in order to achieve accountability. Instead of the general description of flow-net in our previous work, the entire work in this paper is based on the newly proposed formal definition of flow-net.

\section{Definition of Multi-Resolution Flow-Net}

In this section, we propose the formal definition of multiresolution flow-net, and based upon this, we find a solution for event tracing under a few of assumptions.

\subsection{Formal definition of flow-net}

In flow-net, a flow is an entity's event list with time stamps [1]. Each entity (e.g., a file, a TCP segment, an IP packet, or a process) has its event list that logs the entity's events with time stamps. An interconnection of two flows indicates an event was taking place [1]. When using non-relation logging, an administrator may check the log to find out the two events among numerous events, and then infer the relations between the two events. When using flow-net, the flow in the flow-net directly implies to the administrator or other applications the relation between the two events. Since a flow records all the events taking place on an entity, we can use flow-net to trace the events along their relations in the flow. Therefore, by using flow-net, each entity's events and the relations among the events are recorded and thus the entire system's accountability is assured in terms of accountable logging.

A flow-net is denoted by a directed graph $f n=(E, R)$ in which $E$ is the set of nodes and in which $R$ is the set of edges. In the graph, a node $e$ represents an event, and a directed edge $r_{1,2}=\left(e_{1}, e_{2}\right)$ represents the relation that Event $e_{1}$ causes Event $e_{2}$ to take place in a flow. Since a flow is a set of events and relations, we can represent a flow as a series of relations: $f=\left\{r_{1,2}, r_{2,3}, \ldots r_{n-1, n}\right\}$ in which $r_{i, i+1}=\left(e_{i}, e_{i+1}\right)$. If $r_{i, i+1}=\left(e_{i}, e_{i+1}\right)$, then we call $e_{i}$ and $e_{i+1}$ two consecutive events or call $e_{i+1} e_{i}$ 's next event. In this case, $e_{i}$ is called the preceding event, and $e_{i+1}$ is called a successive event. These denotations can be used to describe part of a flow starting from Event $e_{1}$ to Event $e_{n}$. For convenience, we use "flow" as an abbreviation of a part of flow in this paper. Each event and flow has certain properties (i.e., timestamp, event name, flow name, event type).

The multi-resolution feature of an image means that we can zoom in the image to a higher resolution in order to see the particular area that we are interested in for more details, and we can also zoom out of the image to a lower resolution in order to get a comprehensive view of the image. A multiresolution flow-net is a flow-net with multi-layers, and it has the zoom feature that is able to show events with a different resolution.

The lower layer events that are caused by the higher layer event and the flows that connect these lower layer events compose the scope of the higher layer event. The higher layer event is called the parent event, and the lower layer events in the parent event's scope are called scope events. The part of a flow that is composed by these scope events is called the scope flow. We denote the Scope Set as $S_{e}=\left\{r_{1,2}, r_{2,3}, \ldots, r_{i, j}, \ldots, r_{n-1, n}\right\}$ in which $r_{i, j}$ means that Event $e_{i}$ and $e_{j}$ are two consecutive scope events in the same flow. Therefore, Events $e_{1}, e_{2}, \ldots, e_{n}$ are all of Event $e$ 's scope events.

Unlike Relation Set $R$ that is the set of relations in a flow within a layer, Event $e$ 's Scope Set $S_{e}$ is the set of cross-layer relations. Relation Set $R$ denotes the intra-layer relation, and Scope Set $S_{e}$ denotes an inter-layer relation. A multi-resolution flow-net is denoted as $f n=(E, R, S)$ in which $E$ is the set of events, $R$ is the set of relations in a flow within a layer, and $S$ is the set of all the scope sets in this flow-net.

\subsection{Mathematical description of multi-resolution flow-net}

An upper layer event possibly causes multiple lower layer events. For example, in a TCP/IP network, an application layer event "sending a message" will trigger multiple TCP events, including "send segment," "receive ACK", etc.. Similarly, a TCP event may cause multiple network layer events. A multiresolution flow-net logs events and relations on multi-layers, and with the zoom feature, it is able to show events with a different resolution in order to achieve multiple degrees of accountability.

Fig. 1 shows a sample multi-resolution flow-net in which there are three layers. On Layer 1, Flow $f_{11}=\left\{r_{11,12}, r_{12,13}\right\}$. Event $e_{11}$ is an intersection of Flow $f_{10}$ and $f_{11}$. Similarly, we can denote the flows on Layer 2 (i.e., $f_{21}$ and $f_{22}$ ) and the flows on Layer 3 (i.e., $f_{31}, f_{32}, \ldots, f_{3 \mathrm{i}}$ ). Imaging this figure as the multi-layer TCP/IP model helps understand the concept that one event in a higher layer may cause multiple events in a lower layer.

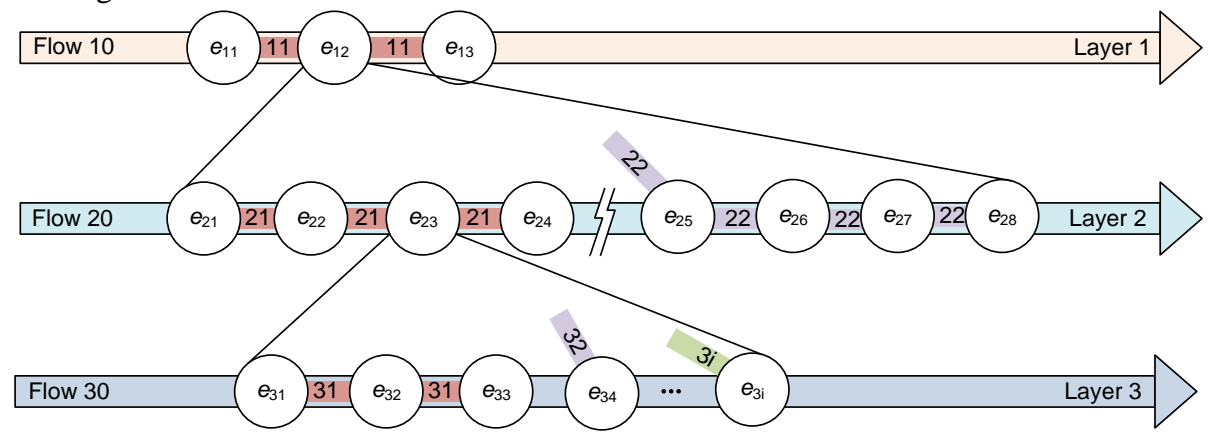


Fig. 1 A sample multi-resolution flow-net. The flow-net in this figure is defined by three parts: (1) the events, which are denoted by the circles; (2) the flows, which are denoted by the strips; and (3) the scopes, which are denoted by the tree-like structures from an upper layer to a lower layer. An event is an intersection of two flows. A flow is composed of events. For example, on Layer 1, Flow $f_{11}=\left\{r_{11,12}, r_{12,13}\right\}$ is formed by three events, $e_{11}, e_{12}$, and $e_{13}$. Event $e_{11}$ is an intersection of Flow $f_{10}$ and $f_{11}$. The events of Flow $f_{21}$ and $f_{22}$ on Layer 2 are caused by Event $e_{12}$ on Layer 1 . With the multi-resolution feature, we can either check out Event $e_{12}$ only, or "zoom in" to Layer 2 to check out the events on Flow $f_{21}$ and $f_{22}$. The events on Flows $f_{21}$ and $f_{22}$ are the scope events of Event $e_{12}$.

The events of Flows $f_{21}$ and $f_{22}$ on Layer 2 are caused by Event $e_{12}$ on Layer 1 . With the multi-resolution feature, we can either check out only Event $e_{12}$ or zoom in to Layer 2 to check out the events on Flows $f_{21}$ and $f_{22}$. The events of Flows $f_{21}$ and $f_{22}$ are the scope events of Event $e_{12}$. The Scope Set of Event $e_{12}$ can be denoted as $S_{e_{12}}=\left\{r_{21,22}, r_{22,23}, r_{23,24}, r_{25,26}, r_{26,27}, r_{27,28}\right\}$. Note that there is not a $r_{24,25}$ in the above set because Event $e_{24}$ and $e_{25}$ belong to two different flows and because $e_{24}$ and $e_{25}$ are not consecutive events. Similarly, we have $S_{e_{23}}$ which is the Scope Set of Event $e_{23}$.

The flow-net in Fig. 1 can be denoted as

$$
\begin{aligned}
f n= & \left(\left\{e_{11}, \cdots, e_{21}, \cdots, e_{31}, \cdots\right\},\right. \\
& \left\{r_{11,12}, \cdots, r_{21,22}, \cdots, r_{31,32}, \cdots\right\}, \\
& \left.\left\{S_{e_{12}}, S_{e_{23}}\right\}\right) .
\end{aligned}
$$

\subsection{Event tracing time cost of multi-resolution flow-net}

We can analyze the time cost for retrieving the flow-net records. Flow-net stores the relations of the events, and thus, we can trace the events by these relations in the flow-net record We assume that the flow-net records for a series of contiguous events in the same layer are stored in a contiguous block of memory or external storage. Therefore, tracing from an event record to the successive one costs a fixed amount of time. However, the records for the events of a different layer may be stored separately.

The time cost caused by zooming from a parent event at a higher layer $i$ to a scope event at a lower layer $j$ is called Zoom In Time Cost, and it is denoted as $T_{i, j}$. The time cost caused by finding an event's parent event from a lower layer $j$ to a higher layer $i$ is called the Parent Tracing Time Cost, and it is denoted as $t_{j, i}$. The space cost that is caused by storing and maintaining the flow-net in order to finding an event's parent event from a lower layer $j$ to a higher layer $i$ is called the Parent Tracing Space Cost, and it is denoted as $s_{j, i}$. All these costs are affected by the specific data structure design of flow-net.

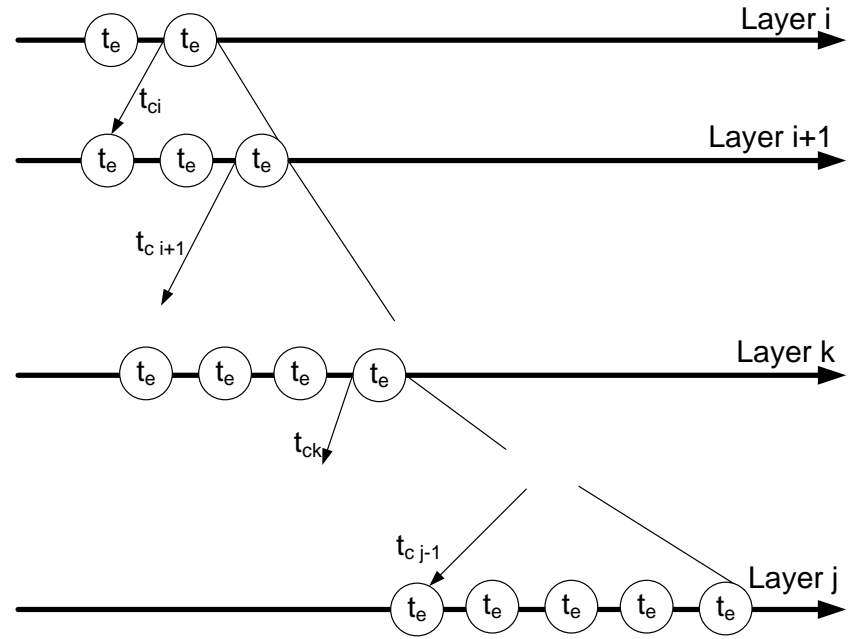

Fig. 2 Time cost of flow-net for tracing all of the flow-net event records

Fig. 2 shows the logical structure of calculating zoom in time cost of flow-net records. Based on our experience from previous implementation/simulation of flow-net, two consecutive events in a relation mostly locate next to each other in the flow-net log, if the two events are within the same layer. This is due to the nature that log items are generated in the time sequence. In most cases, tracing from one event to the successive one in the log takes similar amount of time. Therefore, we assume that the time cost for retrieving a record by tracing from the preceding event to the successive one in the same layer is a fixed amount of time, denoted as $t_{e}$. This is the time cost for intra-layer event tracing. The time cost for tracing from a higher layer event record to the adjacent lower layer one may cost a different amount of time because the records may be stored in a different block of an external storage. This is the time cost for inter-layer tracing. We define $t_{c_{k}}$ as the time cost for tracing an event record from Layer $k$ to its adjacent lower Layer $k+1$. However, if we do a large amount of times of this cross-layer tracing, we will get an average value of $t_{c_{k}}$ for a different $k$. Therefore, we assume a fixed value for each $t_{c}=t_{c_{k}}$ for any $k$. We define $n_{k}$ as the number of event records that we have traced at Layer $k$ and $N$ as the total number of events being traced through all the layers. Therefore, in the case of multi-resolution, the Zoom In Time Cost is

$$
\begin{aligned}
T_{i, j} & =n_{i} \times t_{e}+t_{c_{i}}+n_{i+1} \times t_{e}+t_{c_{i+1}}+\ldots \\
& +n_{j-1} \times t_{e}+t_{c_{j-1}}+n_{j} \times t_{e} \\
& =\sum_{k=i}^{j} n_{k} \times t_{e}+\sum_{k=i}^{j-1} t_{c_{k}}=N t_{e}+(j-i) t_{c} .
\end{aligned}
$$

Therefore, the total time cost for tracing all of the related flow-net event records is proportional to the number of related records. 


\section{Designs of multi-resolution flow-net}

In this section, we first propose two designs for the data structure of multi-resolution flow-net. Based upon these two designs, we then propose a general design scheme for multiresolution flow-net. Then, we define and evaluate the performance of different multi-resolution flow-net designs, and thus, we can find the optimal design.

\subsection{Two designs of the multi-resolution flow-net}

There are three kinds of relations between two events in multi-resolution flow-net: one event causes a successive event taking place in the same flow; one event is a lower layer event's parent event; one event is a higher layer event's scope event. One detailed event and relation recording method was proposed in [22] as our previous work. Moreover, we could have different designs for the flow-net to record events and relations as well. For example, using a foreign key or column in a database to refer to another tuple can denote the relation between two events. Referring to an ID field in an XML-based file can also denote the relation between two events.

Whatever design we use, the relations are normally denoted as a "reference" between events. We can abstract the relation between two events as a reference, which can be implemented by pointer or reference in programming languages, data field in database systems, etc. If we use the reference in the data structure of a flow-net implementation, then a reference from the parent event to the scope event is called a scope pointer, a reference from the scope event to the parent event is called a parent pointer, and a reference from the preceding event to the successive event in a flow is called a flow pointer. The flow pointer denotes the intra-layer relation, and the scope pointer and parent pointer denote inter-layer relations.

Fig. 3 and Fig. 4 show two designs of the scope of multiresolution flow-net. Table 1 lists the similarities and differences of these two designs. We introduce three kinds of references/pointers in each event's data structure. In both designs, the scope pointer of parent event only points to the start of each flow in the scope. The difference between these two designs is the placement of the parent pointer: the first design places a parent pointer in each scope event while the second one places the parent pointer only in the last event of the scope flow.

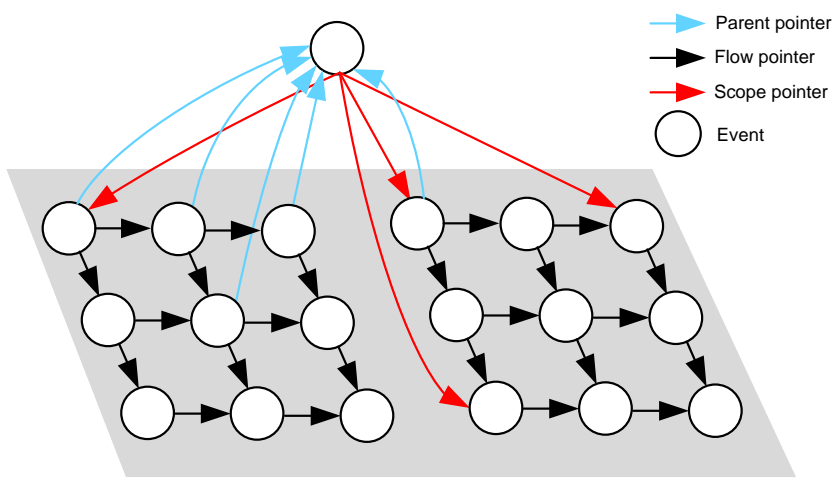

Fig. 3 A design of multi-resolution flow-net data structure for scope: a parent pointer in each scope event

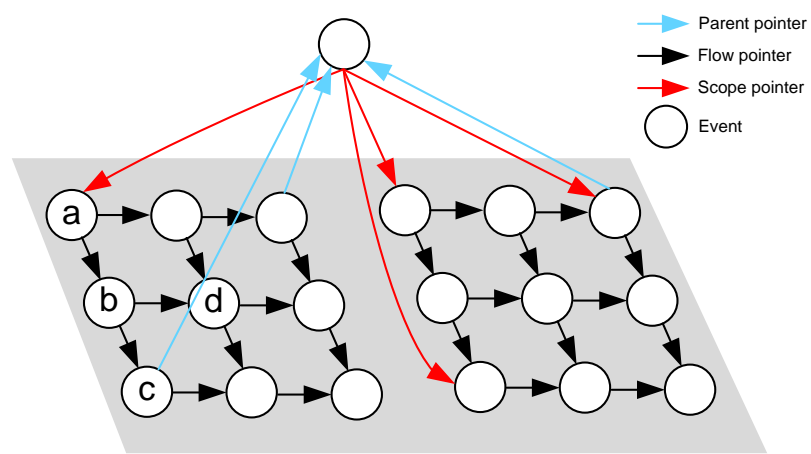

Fig. 4 Another design of multi-resolution flow-net data structure for scope: a parent pointer only in the last scope event

TABLE 1: THE SIMILARITIES AND DIFFERENCES OF THE TWO DESIGNS

\begin{tabular}{|l|l|l|}
\hline & Design in Figs. 3 and 5(a) & Design in Figs. 4 and 5(b) \\
\hline Flow pointer & $\begin{array}{l}\text { In same layer. } \\
\text { From preceding event to successive event. }\end{array}$ \\
\hline Scope pointer & $\begin{array}{l}\text { Cross layers. } \\
\text { From higher layer's parent event to first event in lower } \\
\text { layer. }\end{array}$ \\
\hline Parent pointer & $\begin{array}{l}\text { Cross layers. } \\
\text { From each lower layer } \\
\text { event to higher layer } \\
\text { event. }\end{array}$ & $\begin{array}{l}\text { Cross layers. } \\
\text { From last lower layer event } \\
\text { to higher layer event. }\end{array}$ \\
\hline
\end{tabular}

Fig. 3 and Fig. 5(a) show the first design scheme. As stated before, the parent event's scope pointer points to the start of each flow in the scope. In this design, each scope event has a parent pointer to the parent event.

For any event in flow-net, it must be the crossover of two flows. This means that an event record should have two flow pointers that point to the two successive events in the two flows, respectively. Note that a series of flow pointers on a flow can represent the flow.

For an event record in the higher layer, it has scope pointers pointing to scope event records in the lower layer. In the design shown in Fig. 3, the scope pointers only point to the first event of each flow in the scope at the lower layer.

For each event in the lower layer, it has a parent pointer pointing to its parent event in the higher layer. Some of the scope and parent pointers are not drawn in Fig. 3 due to the space limitations.

Fig. 4 and Fig. 5(b) show another design scheme of the scope of flow-net. In this design, the parent pointer only exists in the last event of a scope flow. Some of the scope and parent pointers are not drawn in Fig. 4 due to the space limitation. In both of the two designs, the three kinds of pointers along with the events intelligibly represent the multi-resolution flow-net.

In the second design, in order to find an event's parent event, we have to trace along the flow pointers and then get the parent pointer from the last event in the flow. This design saves more space than the first design, but it costs more time for an event in a flow to find a parent pointer at the end of the flow. Because each event is the crossover of two flows, we need to decide which flow we are at when tracing on the flow. For example, in Fig. 4, we can find Event $a$ 's parent event by tracing the flow $a-b-c$. Therefore, at Event $b$ we need to ensure 
that we are tracing to Event $c$ but not $d$ in order to get the parent pointer on Event $c$. The similar process for choosing the next event of the flow must take place on all of the events in the flow since each event is the crossover of this flow and another flow.

However, the scopes of two events do not overlap with each other. One parent event causes certain events among the parent's scope while another parent has its different scope events. These two scopes cannot possibly overlap with each other because the two parent events cause different sets of events at the lower layer. This property of multi-resolution flow-net implies that we just need to trace to any event to reach the last event of any scope flow in order to get the parent event. Rather than ensuring the trace to Event $c$ but not Event $d$ in the previous example, we can trace to either of them in order to reach the last event of any scope flow.

The difference between these two designs is the placement of the parent pointer. The first design maintains a parent pointer in each event record while the second design maintains the parent pointer only in the last event record in the scope flow. Both designs use a scope pointer at the parent event to get the start

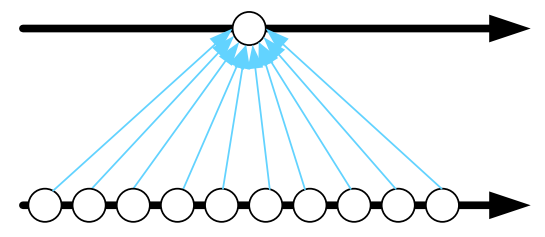

(a)

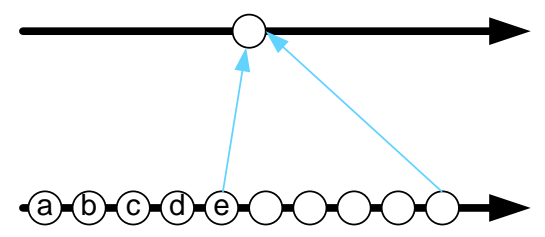

(c) event of its scope flow. Therefore, the first design costs constant time to trace to one event's parent event, and the second design costs linear time to trace the parent event according to the distance from the current event to the last event in the flow. Although the first design saves time for parent tracing, it costs space to save the parent pointers on each event.

\subsection{A general design scheme for multi-resolution flow-net}

The two designs in the previous subsection use the most and least numbers of possible parent pointers, respectively. We could also design schemes that have numbers of pointers in between the two designs.

To trace an event's parent event, we could provide a parent pointer $a$ ) at each event, $b$ ) only at the end event of a flow, or $c$ ) at every certain number of events. Therefore, in a scope, the number of parent pointers can be as large as the number of events in the scope, as small as the number of flows in the scope, or in between.

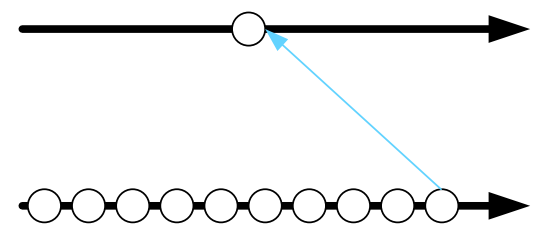

(b)

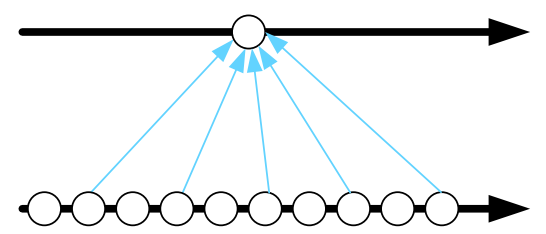

(d)

Fig. 5 Four specific designs for parent pointers

Fig. 5(a) shows the design that has a parent pointer at each event, Fig. 5(b) shows the design that has a parent pointer only at the last event of the scope flow, and Figs. 5(c) and (d) shows the designs that have a parent pointer at some of the events on the flow and they are the cases in between the two extreme cases of Figs. 5(a) and (b). We analyze the performance of these designs in the following subsections.

\subsection{Performance analysis of the multi-resolution flow-net designs}

Therefore, the number of parent pointers on the scope flow can be between 1 to the number of events on the flow. This gives us a general design scheme in which the specific number of parent pointers on the scope flow denotes a specific design. Let $n$ be the number of events in a higher layer event's scope, $m(n \geq m)$ be the number of parent pointers in this scope. We define pointer ratio of this scope as $p=m / n$. For simplicity, we assume that the values of $p$ at any scope on Layer $k$ are the same. We define $m_{k}$ as the number of parent pointers at Layer $k$ and $n_{k}$ as the number of events at Layer $k$. On Layer $k$, the pointer ratio is $p_{k}=m_{k} / n_{k}$. Therefore, we use the value of $p_{k}$ $\left(0 \leq p_{k} \leq 1\right)$ to describe the character of a design. In other words, we can use a different value of $p_{k}$ to differentiate different designs.

Now, we consider the space cost for maintaining the parent pointers in multi-resolution flow-net. As shown in Fig. 5, there are two kinds of event records: the record with a parent pointer and the record without a parent pointer. The former costs more space than the latter. We may put a NULL value at the parent value position in the event record that has no parent pointer. Therefore, we can use a uniform format for both kinds of records, and a NULL value indicates that there is not a parent pointer. However, many modern database systems, such as Oracle Database and SQL Server 2008 and later, handle the NULL value as a variable value so that the first kind of records 
costs more space than the second. Using logging systems has a similar effect.

For an Event $e$ at Layer $j$, if it has a parent pointer, we can trace along this parent pointer to get its parent event at Layer $j$ 1. If Event $e$ does not have a parent pointer, we can trace along the flow pointers until reaching an event with a parent pointer, and then, we can trace along the parent pointer to get the parent event at Layer $j-1$. Repeating this process recursively, we can reach Event $e$ 's grandparent, great grandparent, and other ancestors who are all called parents for convenience. Therefore, we can recursively trace to $e$ 's parent at Layer $i$, where $i<j$. In other words, if we have an Event $e$ at Layer $j$ and we try to trace $e$ 's ancestor at Layer $i$, we can call this ancestor as $e$ 's parent at Layer $i$. We will define and analyze the space and time cost for tracing an event's ancestor/parent.

We assume that the space cost of an event record without the parent pointer is a fixed amount of value that is denoted by $s_{e}$. This can be considered as the space cost for intra-layer event tracing. The space cost of an event record with the parent pointer that is denoted by $s_{c}$, is the summation of $s_{e}$ and parent pointer space cost. This can be considered the space cost for inter-layer tracing. Denote $N$ as the total number of events being traced through all the layers. We have the Parent Tracing Space Cost from Layer $j$ to Layer $i$

$$
\begin{aligned}
s_{j, i} & =\sum_{k=i+1}^{j}\left[\left(n_{k}-m_{k}\right) s_{e}+m_{k} s_{c}\right]=\sum_{k=i+1}^{j}\left[\left(n_{k}-n_{k} p_{k}\right) s_{e}+n_{k} p_{k} s_{c}\right] \\
= & \sum_{k=i+1}^{j} n_{k}\left[\left(1-p_{k}\right) s_{e}+p_{k} s_{c}\right]=N \sum_{k=i+1}^{j}\left[\left(1-p_{k}\right) s_{e}+p_{k} s_{c}\right] .
\end{aligned}
$$

If we assume that for any $k$ that the pointer ratio $p_{k}$ is a real number $p$ and $0 \leq p \leq 1$, then the value of $p$ determines the character of a design. Because each value of $p$ corresponds to a design, we use $p$ to differentiate the designs. We have

$$
\begin{aligned}
s_{j, i} & =N \sum_{k=i+1}^{j}\left[\left(1-p_{k}\right) s_{e}+p_{k} s_{c}\right]=N \sum_{k=i+1}^{j}\left[(1-p) s_{e}+p_{S_{c}}\right] \\
& =N(j-i)\left[(1-p) s_{e}+p_{S_{c}}\right]=N(j-i)\left[p\left(s_{c}-s_{e}\right)+s_{e}\right] .
\end{aligned}
$$

Now, we consider the time cost for maintaining the parent pointers in multi-resolution flow-net. The time cost for retrieving a record by tracing from a lower layer event record to the adjacent higher layer one may cost a different amount of time because the records may be stored in a different block of an external storage. We have the worst Parent Tracing Time Cost from Layer $j$ to Layer $i$

$$
t_{j, i}=\sum_{k=i+1}^{j}\left(\frac{n_{k}-m_{k}}{m_{k}} t_{e}+t_{c_{k}}\right)=\sum_{k=i+1}^{j}\left(\frac{n_{k}}{m_{k}} t_{e}+t_{c_{k}}\right)-(j-i) t_{e} .
$$

If we assume for any $k, t_{c}=t_{c_{k}}$, then we have

$$
\begin{aligned}
t_{j, i} & =\sum_{k=i+1}^{j}\left(\frac{n_{k}}{m_{k}} t_{e}+t_{C_{k}}\right)-(j-i) t_{e}=\sum_{k=i+1}^{j}\left(\frac{n_{k}}{m_{k}} t_{e}+t_{c}\right)-(j-i) t_{e} \\
& =\sum_{k=i+1}^{j} \frac{n_{k}}{m_{k}} t_{e}+(j-i)\left(t_{c}-t_{e}\right)=\sum_{k=i+1}^{j} t_{e} / p_{k}+(j-i)\left(t_{c}-t_{e}\right) .
\end{aligned}
$$

If we assume for any $k$ that the pointer ratio $p_{k}$ is a constant value $p$, then we have

$$
\begin{aligned}
t_{j, i} & =\sum_{k=i+1}^{j} t_{e} / p_{k}+(j-i)\left(t_{c}-t_{e}\right)=(j-i) t_{e} / p+(j-i)\left(t_{c}-t_{e}\right) \\
& =(j-i)\left[(1 / p-1) t_{e}+t_{c}\right] .
\end{aligned}
$$

In summary, each value of $p$ corresponds to a design and affects the value of both $s_{j, i}$ and $t_{j, i}$.

\subsection{Optimal design for multi-resolution flow-net}

Both $s_{j, i}$ and $t_{j, i}$ affect the performance of the design, and they are determined by the pointer ratio $p$. It is a negative correlation between $t_{j, i}$ and $p$ while it is a positive correlation between $s_{j, i}$ and $p$. In this subsection, we give the definition of performance for a design, discuss the correlation between the performance and $p$, and find the optimal value of $p$.

We can consider $s_{j, i}$ and $t_{j, i}$ separately. For a given value $V_{0}$, if we require the Parent Tracing Space Cost $s_{j, i}<V_{0}$, then we have $\quad N(j-i)\left[p\left(s_{c}-s_{e}\right)+s_{e}\right]<V_{0} \quad$. Therefore, $p<\frac{V_{0} /[N(j-i)]-s_{e}}{s_{c}-s_{e}}$. For a given value $W_{0}$, if we require the Parent Tracing Time Cost $t_{j, i}<W_{0}$, then we have $(j-i)\left[(1 / p-1) t_{e}+t_{c}\right]<W_{0}$. Therefore, $p>\frac{t_{e}}{W_{0} /(j-i)-t_{c}+t_{e}}$

Now, we get the valid interval of $p$ as $\frac{t_{e}}{W_{0} /(j-i)-t_{c}+t_{e}}<p<\frac{V_{0} /[N(j-i)]-s_{e}}{s_{c}-s_{e}}$. However, this interval could be empty.

Therefore, we obtain an interval of $p$ under the separate requirement of space and time cost. However, an optimal value of $p$ has not been deduced.

Furthermore, we can consider $s_{j, i}$ and $t_{j, i}$ together in order to obtain an optimal value of $p$. We define a weighting parameter $0 \leq \alpha \leq 1$ and let $\beta=1-\alpha$, and then, define the resource cost of design $p$ as

$r_{c}(p)=\alpha_{s_{j, i}}+\beta t_{j, i}$

$=\alpha N(j-i)\left[p\left(s_{c}-s_{e}\right)+s_{e}\right]+\beta(j-i)\left[(1 / p-1) t_{e}+t_{c}\right]$,

where $N, j, i, s_{c}, s_{e}, t_{c}$, and $t_{e}$ can all be viewed as constant values. To get the minimum value of $r_{c}(p)$, we have to get the value of $p$ such that the derivative of $r_{c}(p)$ equals 0 . We have $r_{c}^{\prime}(p)=\alpha N(j-i)\left(s_{c}-s_{e}\right)-\beta(j-i) t_{e} / p^{2}$, and $r_{c}^{\prime \prime}(p)=2 \beta(j-i) t_{e} / p^{3}>0$. If we require $r_{c}^{\prime}(p)=0$, We have $p=\sqrt{\frac{\beta_{t_{e}}}{\alpha N\left(s_{c}-s_{e}\right)}}$, which is the optimal value of $p$ and makes $r_{c}(p)$ minimum. This optimal value is for general cases in which the ratio between $\alpha$ and $\beta$ is set.

However, one dilemma for us to determine the value of $p$ is that it depends on $N$. This means that we have to know the number of events before we determine the pointer ratio. Given the fact that flow-net tends to cost a large amount of space to record the events and relations no matter how we design it, we can consider the time cost more than the space cost. Therefore, we can solve the problem by setting $\beta=\alpha N$. Since $\beta=1-\alpha$, 
we have $\alpha=1 /(N+1)$. This means when considering the resource cost of flow-net, we focus on the time cost rather than the space cost. Therefore, $p=\sqrt{t_{e} /\left(s_{c}-s_{e}\right)}$ is the optimal value of $p$ and makes the $r_{c}(p)$ the minimum, if $\alpha=1 /(N+1)$. Note that this optimal value is for a special case in which the ratio between $\beta$ and $\alpha$ is set to be $N$.

Therefore, the optimal value of $p$ does not depend on the layer number $i$ or $j$, and this means that we can set the pointer ratio $p$ to the same value for all layers. Moreover, $p$ depends on the inter-layer tracing space cost $s_{c}$, but it does not depend on the inter-layer tracing time cost $t_{c}$.

\section{Flow-net Used in TCP/IP Networks}

In this section, we discuss on how to deploy flow-net in actual TCP/IP networks by checking out some well known TCP/IP protocols and giving the flow-net event lists of these protocols. In this paper, we call the behaviors that are defined in the TCP/IP protocols as "protocol events", and we record them in the flow-net logging. Therefore, these events are actually protocol level events in TCP/IP networks. Our work focuses on finding and summarizing the events that are defined by TCP/IP protocols. Therefore, our goal is not revising the behaviors of the protocols but only adding logging functions for TCP/IP networks through the help of flow-net. By using flow-net, each entity and layer in the network system is held responsible for its own action, and thus, the accountable logging of the entire system is assured.

\subsection{HTTP and HTTPS}

Before looking TCP/IP events, we first check Hypertext Transfer Protocol (HTTP) and HTTP Secure (HTTPS), which two of the most widely used application layer protocols. HTTP is an application protocol for data communication on the World Wide Web. Designing for data exchange, HTTP can be implemented on both servers and clients. The client sends
HTTP request messages to the server by establishing a TCP connection [12]. Upon receiving a request, the server sends back a message with the requested data [12]. The events in HTTP include sending/receiving the request messages and sending/receiving the response messages. A response message normally includes a response head and a body. The Events "sending request" and "receiving response" normally take place at the client, while the events "receive request" and "sending response" normally take place at the server.

HTTPS is a secure communication protocol based upon HTTP. HTTPS makes use of the digital certificate and Certificate Authority (CA) to assure the authentication of the website and to prevent a man-in-the-middle attack and other attacks. In order to use HTTPS, we must assure that a) the browser at the client runs correctly, b) the certificate authority is trustworthy, c) the website provides the correct certificate that matches the website, and d) the data encryption/decryption is trustworthy [13]. Table 2 lists the HTTP and HTTPS events. Due to the space limitation, we only list a part of the events. TABLE 2: HTTP AND HTTPS EVENTS

\begin{tabular}{|l|l|}
\hline Event & Description \\
\hline Send/Receive HEAD request & Must be implemented at server. \\
\hline Send/Receive GET request & Must be implemented at server. \\
\hline Send/Receive POST request & Check if a URL is serviceable, etc. \\
\hline Send/Receive PUT request & Upload certain resource. \\
\hline Send/Receive DELETE request & Deletes certain resource. \\
\hline Send/Receive TRACE request & Echo back the received request. \\
\hline Send/Receive OPTIONS request & Return supported HTTP methods. \\
\hline Send/Receive CONNECT request & Usually used in HTTPS. \\
\hline Send/Receive PATCH request & Apply partial modifications. \\
\hline Send/Receive response & Response the request. \\
\hline Encrypt message & $\begin{array}{l}\text { The server/client encrypts the message } \\
\text { using the symmetric key. }\end{array}$ \\
\hline Send/Receive encrypted message & $\begin{array}{l}\text { Data transmission is secure, because } \\
\text { the message is encrypted by the } \\
\text { symmetric key. The message in this } \\
\text { event may be a specific HTTP event. }\end{array}$ \\
\hline Decrypt message & $\begin{array}{l}\text { The server/client decrypts the message } \\
\text { by using the symmetric key. }\end{array}$ \\
\hline
\end{tabular}

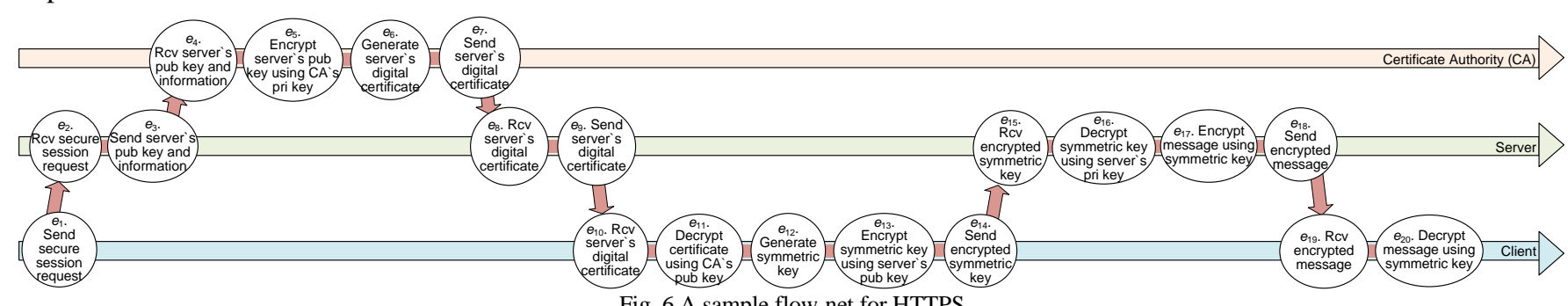

Fig. 6 shows a sample flow-net of an HTTPS connection. CA is a trustworthy third party that issues digital certificates. In this example, the client sends a request to the server for a secure session. After receiving this request, the server contacts the CA to generate the server's digital certificate in order to show the server's authentication to the client. To generate this digital certificate, CA uses its private key to encrypt the server's public key and other information. Then, CA sends the server's digital certificate to the server. After receiving the digital certificate, the server sends it to the client. The client decrypts the digital certificate by using CA's public key in order to get the server's public key. The secure session between the server and the client is encrypted by a symmetric key. This symmetric key is generated at the client and should be sent to the server. In order to secure the symmetric key transmission, the client uses the server's public key, which the client just obtained by decryption, to encrypt the symmetric key. Also, the server can decrypt to get the symmetric key by using the server's private key. In this way, both the server and the client use the same symmetric key, and this symmetric key is 
transmitted in a secure way by using a digital certificate and server's public/private key. And then, the server and the client can use the symmetric key to secure the following messages in transmission. Because the symmetric key is transmitted to the server and encrypted by the server's public key, the symmetric key is not leaked.

We use flow-net to show the events in Fig. 6, and the flownet of this sample HTTPS session is denoted as follow.

$$
f n_{\text {HTTPS }}=\left(\left\{e_{1}, \cdots, e_{20}\right\},\left\{r_{1,2}, \ldots, r_{19,20}\right\}\right) \text {. }
$$

Using flow-net, a HTTP/HTTPS session is denoted integrity. This subsection shows that flow-net is effective to achieve accountable logging in the application Layer. Moreover, flownet can be used for the other layers in TCP/IP networks to achieve accountable logging as well. In the following subsections, we use a similar way to study TCP/IP protocols and draw the flow-net for the data communications using these protocols in order to achieve accountable logging.

\subsection{TCP Events}

Transmission Control Protocol TCP) and User Datagram Protocol (UDP) are two of the most well known transport layer protocols. In this section, we study TCP protocols from the perspective of flow-net, and we also present a sample flow-net of a TCP session. TCP uses a three-way handshake to establish a connection. The TCP sender received data from its application layer and encapsulates the data into segments, which will be sent to the TCP recipient. After receiving the segments, the TCP recipient sends ACKs to the TCP sender, and it extracts data from the segments to the application layer. TCP congestion control, including slow start, congestion avoidance, fast retransmit, and fast recovery, takes place along with segment transmission. Other TCP events are also executed to assist the segment transmission [14-15].

Some TCP events, such as "send ACK," "send segment," and "change window scaling," are indicated by a specific format in the TCP header. Therefore, these events can be represented by the TCP header. Some other TCP events, such as congestion control, are affected by the TCP status and executed at the end hosts. For example, both slow start and congestion avoidance take place after a segment transmission, but slow start requires a congestion window smaller than the threshold, and congestion avoidance requires the opposite. Flow-net records events and their relations but not status (i.e., congestion window size and threshold in this case). However, the events, "change congestion window size" and "change threshold", can be recorded by flow-net. Therefore, we can achieve accountable logging by examining the events and relations recorded by flow-net. Table 3 lists the TCP events in which we classify the events based upon their purposes.

\begin{tabular}{|c|c|c|}
\hline Category & Event & Description \\
\hline \multirow{3}{*}{$\begin{array}{l}\text { Before and } \\
\text { After } \\
\text { Connection }\end{array}$} & Listen & \\
\hline & Connection Established & \\
\hline & Connection Closed & \\
\hline \multirow{3}{*}{$\begin{array}{l}\text { Establish } \\
\text { Connection }\end{array}$} & Send/Receive SYN & \multirow{3}{*}{$\begin{array}{l}\text { These six events constitute a } \\
\text { three-way handshake, which } \\
\text { establishes TCP connection. }\end{array}$} \\
\hline & Send/Receive SYN-ACK & \\
\hline & Send/Receive ACK & \\
\hline Data & Receive data & TCP sender received data \\
\hline
\end{tabular}

\begin{tabular}{|c|c|c|}
\hline \multirow[t]{9}{*}{ transmission } & Application Layer & \multirow{2}{*}{$\begin{array}{l}\text { from Application Layer and } \\
\text { encapsulates the data to } \\
\text { segments, which will send to } \\
\text { the TCP recipient. }\end{array}$} \\
\hline & $\begin{array}{l}\text { Encapsulate data to } \\
\text { segments }\end{array}$ & \\
\hline & Send/Receive segment & \multirow{3}{*}{$\begin{array}{l}\text { Data transmission. } \\
\text { TCP arranges the order of } \\
\text { segments to compose } \\
\text { original data for Application } \\
\text { Layer. }\end{array}$} \\
\hline & $\begin{array}{l}\text { Receive segment: in- } \\
\text { order segment arrival }\end{array}$ & \\
\hline & \multirow{2}{*}{$\begin{array}{l}\text { Receive segment: out-of- } \\
\text { order segment arrival } \\
\text { Send/receive ACK } \\
\end{array}$} & \\
\hline & & \\
\hline & Timeout & \\
\hline & Check checksum & \\
\hline & $\begin{array}{l}\text { Extract data } \\
\text { Application Layer }\end{array}$ & $\begin{array}{l}\text { Extract Application Layer } \\
\text { message from the TCP } \\
\text { segment and send to } \\
\text { Application Layer }\end{array}$ \\
\hline \multirow{6}{*}{$\begin{array}{l}\text { TCP } \\
\text { Congestion } \\
\text { Control }\end{array}$} & Slow start & $\begin{array}{l}\text { Triggered by timeout or } \\
\text { duplicate ACKs. Set } \\
\text { congestion window to be } \\
\text { one segment, and half } \\
\text { threshold. Exponentially } \\
\text { increase congestion window } \\
\text { until reaching threshold. }\end{array}$ \\
\hline & Congestion avoidance & $\begin{array}{l}\text { Triggered when reaching } \\
\text { threshold. Linearly increase } \\
\text { congestion window. }\end{array}$ \\
\hline & Fast retransmit & $\begin{array}{l}\text { Triggered by three duplicate } \\
\text { ACKs. Resend segments. }\end{array}$ \\
\hline & Fast recovery & $\begin{array}{l}\text { Triggered by three duplicate } \\
\text { ACKs. Congestion } \\
\text { window size is reduced to } \\
\text { the slow-start threshold. Fast } \\
\text { recovery executes fast } \\
\text { retransmit followed by } \\
\text { congestion avoidance. }\end{array}$ \\
\hline & $\begin{array}{l}\text { Change congestion } \\
\text { window size }\end{array}$ & $\begin{array}{l}\text { Window size is changed } \\
\text { during slow start or } \\
\text { congestion avoidance. }\end{array}$ \\
\hline & Change threshold & $\begin{array}{l}\text { Caused by congestion. Slow } \\
\text { start must just take place. }\end{array}$ \\
\hline \multirow{3}{*}{$\begin{array}{l}\text { Close } \\
\text { Connection }\end{array}$} & Send/Receive FIN & \multirow{2}{*}{$\begin{array}{l}\text { This handshake is the } \\
\text { regular way to close } \\
\text { connection, by using FIN } \\
\text { segment. }\end{array}$} \\
\hline & Send/Receive FIN-ACK & \\
\hline & Send RST & $\begin{array}{l}\text { Set RST field in TCP header } \\
\text { as } 1 \text { to terminate TCP } \\
\text { connection immediately. }\end{array}$ \\
\hline Flow control & window size changed & $\begin{array}{l}\text { The size of receive window } \\
\text { is used for changing flow } \\
\text { rate. }\end{array}$ \\
\hline \multirow[b]{2}{*}{$\begin{array}{l}\text { Window } \\
\text { scaling }\end{array}$} & window size changed to 0 & Sender stops sending data. \\
\hline & Change window scaling & $\begin{array}{l}\text { An option to increase the } \\
\text { size of TCP receive window. }\end{array}$ \\
\hline Out of band & Urgent data & $\begin{array}{l}\text { Alters the receiver for } \\
\text { urgent data. }\end{array}$ \\
\hline $\begin{array}{l}\text { Forcing } \\
\text { delivery }\end{array}$ & Push data & Send this data immediately. \\
\hline
\end{tabular}

After comprehending the TCP events, we present an example that shows several TCP events. Fig. 7 shows a sample TCP data transmission that Node A sends data to Node B. In this example, Event $e_{1}$ "receive data from the application layer" takes place at Node $\mathrm{A}$, and is followed by Event $e_{2}$ "encapsulate data to segments". After Event $e_{3}$ "send segment seq\# 1", Event $e_{4}$ "Receive segment seq\# 1" on Node B takes place. Similarly, Event $e_{9}$ on Node A and Event $e_{10}$ on Node B take place. After receiving both of these two segments, Node B sends two ACKs to Node A. An event for congestion control, 
$e_{8}$ "Slow start and change congestion window size", takes place after Node B receives the ACK. Actually, Event $e_{8}$ is composed of two events, but we combine it into one event in the figure because of the space limitation. Similarly, two congestion control events $e_{14}$ "change threshold" and $e_{15}$ "Congestion Avoidance and change window size" take place after Node B receives the ACK. A flow in flow-net is composed of a series of events. For example, the events related with the segment seq\# 1 compose Flow $\alpha$, and the events taking place on Node A compose Flow A. An event is an intersection of two flows. After receiving the two segments, Node B also executes Event $e_{14}$ and $e_{15}$ to extract the data from the segments and send the data to the application layer.

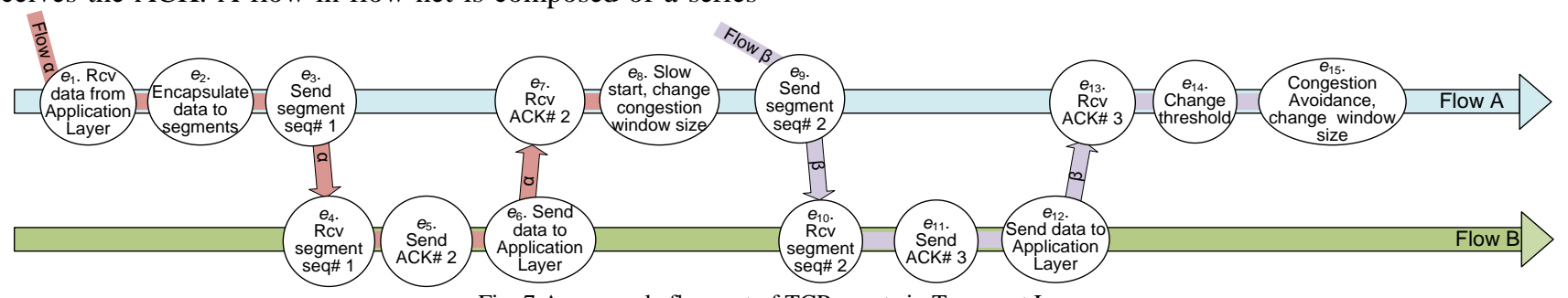

Fig. 7 An example flow-net of TCP events in Transport Layer

The events in the aforementioned example are shown in Fig. 7 , and the flow-net of this sample TCP session is denoted as follow.

$$
f n_{T C P}=\left(\left\{e_{1}, \cdots, e_{15}\right\},\left\{r_{1,2}, \ldots, r_{7,8}, r_{9,10}, \ldots, r_{14,15}\right\}\right) .
$$

\subsection{Other events in TCP/IP networks}

UDP protocol is another important protocol in the transport layer. Unlike TCP, the UDP protocol is a connectionless protocol without the complicated connection establishment and congestion control [16]. Therefore, the number of events in UDP is much fewer than TCP. The network layer is responsible for packet routing through the routers in the network. Internet Protocol (IP) is the principal communications protocol in the network layer while Internet Control Message Protocol (ICMP) is responsible for control purpose and error indicating [17-20]. The data link layer and physical layer are responsible for transferring data between two adjacent network nodes. For example, IEEE 802.11 standards for wireless local area network communication are developed and implemented in both the data link layer and the physical layer [33, 34, 35]. Due to the space limitation, we do not give the sample flow-net for the aforementioned protocols in this subsection.

\section{Multi-Resolution Flow-net in TCP/IP}

In this section, we discuss on how to deploy multi-resolution flow-net in actual TCP/IP networks. The multi-layer feature of TCP/IP networks brings us the chance to achieve multiple degrees of accountability TCP/IP networks. In TCP/IP networks, communications only take place across two adjacent layers. We apply the multi-resolution flow-net to TCP/IP networks and provide designs for distinguishing the scope in TCP/IP networks. By using multi-resolution flow-net, for a low degree of accountability, we can check out an event in a higher layer. For a higher degree of accountability, we can zoom in on this event and trace to this event's scope events in the lower layer. Multiple degrees of accountability in TCP/IP networks therefore correspond to events recorded by flow-net on multiple layers.

In this section, we first give an example of applying multiresolution flow-net in TCP/IP networks, and then we give suggestions for determining a higher layer event's scope events.

\subsection{An example of multi-resolution flow-net in TCP/IP networks}

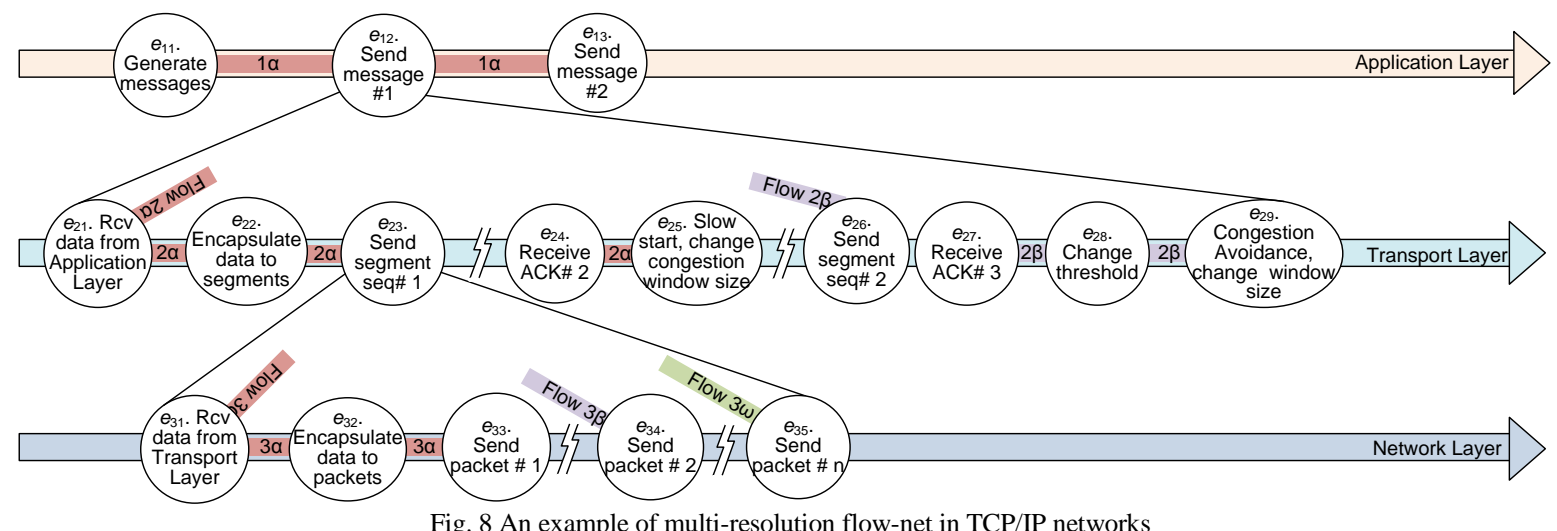

Fig. 8 shows an example of the multi-resolution flow-net applied in TCP/IP networks. Multi-resolution flow-net can be drawn as a tree architecture that maintains the parent and scope events. An application layer event $e_{12}$ "Send message" causes 
multiple TCP events, including the two TCP segment sending events and congestion control events. After executing Event $e_{21}$ "Receive data from Application Layer", the TCP layer generates two segments that are ready to be sent. Events "Encapsulate data to segments", "Send segment seq\# 1," and "Receive ACK\# 2" correspond to sending the first segment. After the TCP layer receives the ACK, an event for congestion control, $e_{25}$ "Slow start and change congestion window size" takes place. Actually Event $e_{25}$ is composed of two events, but we combine them into one event in the figure because of the space limitation. These TCP events compose a flow for the first segment. Similarly, the TCP events for the second segment compose another flow at the network layer. In this example, one event at the application layer causes two flows at the transport layer. All of the above TCP events are the scope events of the application layer Event $e_{12}$.

Fig. 8 also shows the scope events of Event $e_{23}$ "Send segment seq\# 1" at the network layer. The network layer flows correspond to IP packets that are generated by fragmenting the TCP segment to several parts and encapsulating them using an IP header.

The aforementioned example shows that in TCP/IP networks, an event at the upper layer may cause multiple events and even multiple flows at the lower layer. Therefore, multi-resolution flow-net can be applied on TCP/IP network naturally in order to achieve multiple degrees of accountability. To achieve a low degree of accountability, in this example, it is enough for us to check out Event $e_{12}$ "Send message" at the application layer. To achieve a high degree of accountability, it is enough for us to check out Event $e_{12}$ 's scope events (e.g., Event $e_{23}$ "Send segment seq\# 1") at the transport layer. To achieve an even higher degree of accountability, we have to check out the events (e.g., Event $e_{23}$ 's scope events) at the network layer. Besides $e_{23}$, other events at the transport layer have scope events at the network layer as well, but Fig. 8 shows only part of the scope events due to the space limitation. The flow-net in Fig. 8 can be denoted as follows:

$$
\begin{aligned}
& f n_{\text {example }}= \\
& \quad\left\{e_{11}, e_{12}, e_{13}, e_{21}, \cdots, e_{29}, e_{31}, \cdots, e_{35}\right\}, \\
& \quad\left\{r_{11,12}, r_{12,13}, r_{21,22}, r_{22,23}, r_{24,25}, \cdots, r_{31,32}, r_{32,33}, \cdots\right\}, \\
& \left.\quad\left\{S_{12}, S_{23}\right\}\right), \\
& \text { where } S_{12}=\left\{r_{21,22}, \cdots\right\}, S_{23}=\left\{r_{31,32}, \cdots\right\} .
\end{aligned}
$$

\subsection{Suggestions for determining scope events in TCP/IP networks}

In order to apply multi-resolution flow-net on TCP/IP networks, we need to decide how to determine a TCP/IP event's scope events. The principle of the determination is that the lower layer events caused by a higher event should be this event's scope events. We give our suggestions for determining an event's scopes in Table 4. Since there are numerous

\begin{tabular}{|c|c|c|}
\hline $\begin{array}{l}\text { Parent event at } \\
\text { the higher layer }\end{array}$ & Scope events at the lower layer & Description \\
\hline \multirow{6}{*}{ Send message } & Receive data from Application Layer & \multirow{6}{*}{ Using TCP } \\
\hline & Encapsulate data to TCP segments & \\
\hline & Calculate checksum & \\
\hline & Send TCP packet & \\
\hline & Receive ACK & \\
\hline & $\begin{array}{l}\text { TCP Congestion Control events, } \\
\text { including }\end{array}$ & \\
\hline \multirow{4}{*}{ Send message } & Receive data from Application Layer & \multirow{4}{*}{ Using UDP } \\
\hline & Encapsulate data to packet & \\
\hline & UDP sender calculates checksum & \\
\hline & Send Packet & \\
\hline \multirow{7}{*}{$\begin{array}{l}\text { Receive } \\
\text { message }\end{array}$} & Receive segment & \multirow{7}{*}{ Using TCP } \\
\hline & $\begin{array}{l}\text { Receive segment: in-order segment } \\
\text { arrival }\end{array}$ & \\
\hline & $\begin{array}{ll}\text { Receive segment: } & \text { out-of-order } \\
\text { segment arrival } & \\
\end{array}$ & \\
\hline & Check checksum & \\
\hline & Extract data to Application Layer & \\
\hline & Send ACK & \\
\hline & TCP Congestion Control events & \\
\hline \multirow{4}{*}{$\begin{array}{l}\text { Receive } \\
\text { message }\end{array}$} & Receive Packet & \multirow{4}{*}{ Using UDP } \\
\hline & Extract data from packet & \\
\hline & UDP sender checks checksum & \\
\hline & Send data to Application Layer & \\
\hline \multirow{4}{*}{$\begin{array}{l}\text { Send TCP } \\
\text { segment } \\
\text { / Send TCP } \\
\text { ACK } \\
\text { / Send UDP } \\
\text { packet }\end{array}$} & Receive data from Transport Layer & \multirow{4}{*}{$\begin{array}{l}\text { IP events are } \\
\text { the scope } \\
\text { events }\end{array}$} \\
\hline & Encapsulate data to IP segments & \\
\hline & Checksum & \\
\hline & Send IP packet & \\
\hline \multirow{4}{*}{$\begin{array}{l}\text { Receive TCP } \\
\text { segment } \\
\text { / Receive TCP } \\
\text { ACK } \\
\text { / Receive UDP } \\
\text { packet }\end{array}$} & Receive IP packet & \multirow{4}{*}{$\begin{array}{l}\text { IP events are } \\
\text { the scope } \\
\text { events }\end{array}$} \\
\hline & Checksum & \\
\hline & Extract data to Transport Layer & \\
\hline & Send data to Transport Layer & \\
\hline
\end{tabular}
protocols implemented in TCP/IP networks, we only list part of the suggested scope event determination in Table 4 due to the space limitation.

TABLE 4 SUGGESTIONS OF MULTI-RESOLUTION SOLUTION FOR TCP/IP

\section{Performance Evaluation}

In this section, we present the evaluation results for the proposed multi-resolution flow-net. We first evaluate the Zoom In Time Cost. Simulations on tracing from an upper layer event to lower layer events are present as well. The simulation programs for building a flow-net system in which a large amount of events and their relations are maintained were written in Java language. The time cost generated in the experiments was measured by the number of time slots. 


\subsection{Evaluation of Zoom In Time Cost}

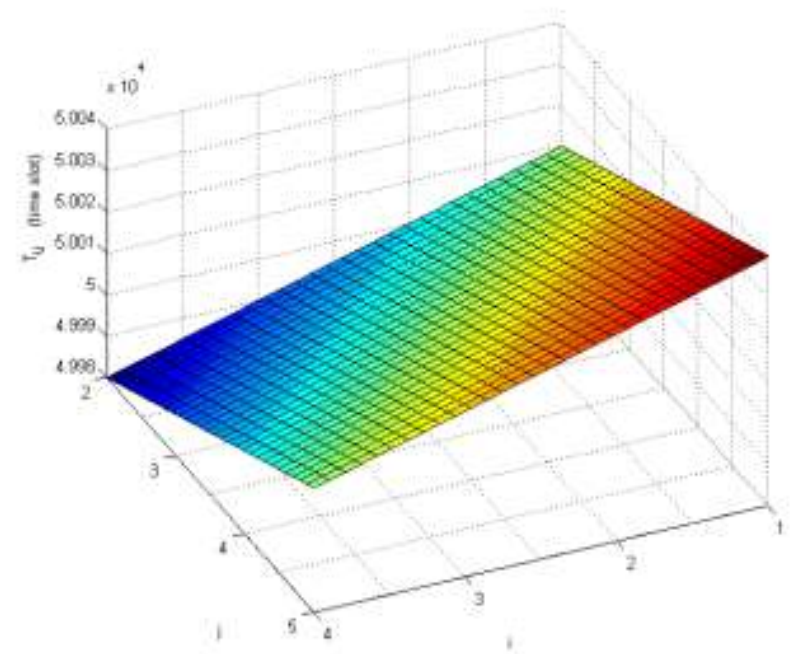

Fig. 9 Tracing time from Layer $i$ to $j$. The unit of the vertical axis is time slot.

In Section 3, we define the Zoom In Time Cost $T_{i, j}$ as the time cost caused by tracing from a parent event at a higher layer $i$ to a scope event at a lower layer $j$. Fig. 9 shows the evaluation results for a multi-resolution flow-net system, which has five layers. The intra-layer event tracing time $t_{e}$ and the inter-layer event tracing time $t_{c}$ re set to be two constant values. In Fig. 9, the $\mathrm{x}$-axis and $\mathrm{y}$-axis represent $i$ and $j$, respectively, and the z-axis represents the value of $T_{i, j}$. Fig. 9 shows that for a given $i$, a larger $j$ causes a larger $T_{i, j}$ because the tracing process crosses more layers and consumes more time than that with a smaller $j$. For a given $j$, a smaller $i$ leads to a larger $T_{i, j}$ because the tracing process crosses more layers and consumes more time than that with a larger $i$. Therefore, the larger the difference between the values of $i$ and $j$ indicates that there is a larger $T_{i, j}$.

\subsection{Performance evaluation of the multi-resolution flow- net designs}

In this subsection, we also present simulation results that calculate the resource cost $r_{c}(p)$ for different values of $p$. We use the values of $p$ to differentiate the designs and to calculate the resource cost $r_{c}(p)$ of each design. We set $1 \leq i<j \leq 5$. The values of intra-layer event tracing time $t_{e}$, inter-layer event tracing time $t_{c}$, intra-layer event tracing space $s_{e}$, and inter-layer event tracing space $s_{c}$ are all set to be certain constant values.

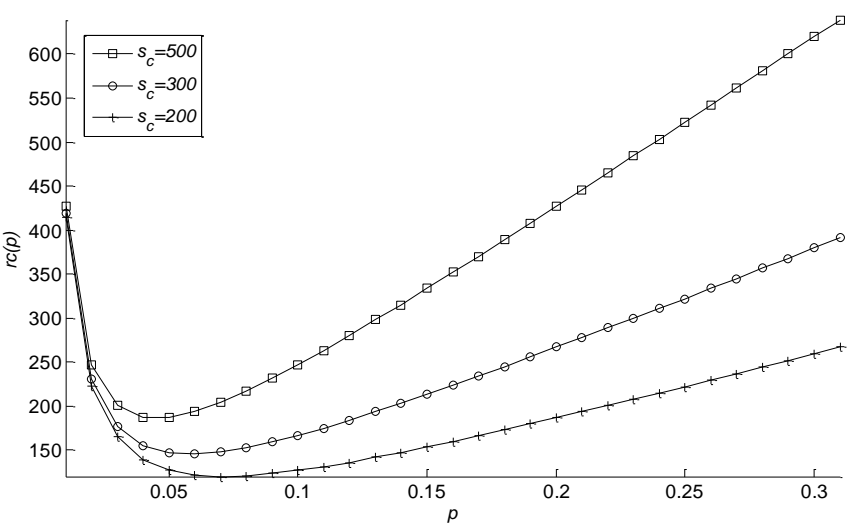

Fig. 10 Resource cost when $\beta=N \alpha$

However, the value of the weighting parameters $\alpha$ and $\beta$ are set in a different way. In Fig. 10 , we set $\beta=N \alpha$ in which $N$ is the total number of events being traced through all the layers. The curves in Fig. 10 correspond to $s_{c}=200,300$, and 500, respectively. Fig. 10 verifies that the minimum value of $r_{c}(p)$ is at $p=\sqrt{t_{e} /\left(s_{c}-s_{e}\right)}$ when $\beta=N \alpha$.

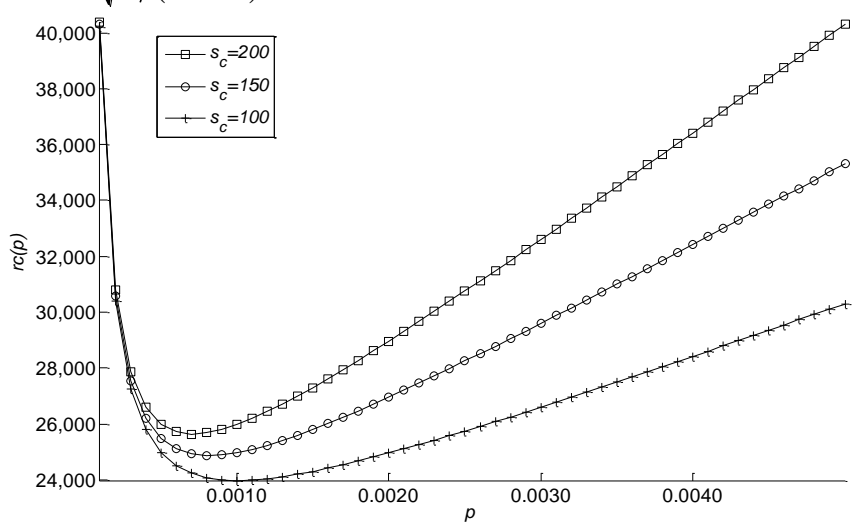

Fig. 11 Resource cost when $\alpha=\beta$

In Fig. 11, we set $\alpha=\beta$, and the results show that the $\mathrm{x}$ coordinate that yields to the minimum value of $r_{c}(p)$ depends on the value of $p$. Since $N$ is a very large value in the flow-net, no matter how we set the other parameters, the $\mathrm{x}$-coordinate that yields to the minimum value of $r_{c}(p)$ is usually very close to 0 . Both Fig. 10 and Fig. 11 show that the value of $r_{c}(p)$ does not change sharply when the value of $p$ is approaching to 1 . This implies that when we are not sure about the setting of the parameters in the implementation of flow-net, a smaller value of $p$ is more notable than other parameters. Therefore, we can set $p$ as a small value and make our design adapt to the other parameters, and then, we can gradually increase $p$ in order to get the minimum value of $r_{c}(p)$.

\subsection{Simulation of event tracing}

With multi-resolution flow-net logging, a lower layer event can be found by tracing from the parent to the scope event and repeating the same process. Therefore, in a few steps, a given event in the lower layer (called target event) can be found through the event relations maintained by multi-resolution flow-net. Non-relation logging does not record the relations between events or maintain the multi-resolution feature. To 
find the target event in the non-relation logging log, we have to scan all the events taking place after the application message receives the event. In this subsection, we simulate the above process and compare the time of finding the target event using flow-net and non-relation logging.

In the simulation for flow-net logging, a log file that records events and relations and maintains the multi-resolution feature is generated. We set the flow-net to record four layers of events The scope pointer (across two layers from parent event to child event) and flow parent (from preceding event to successive event in a flow) are maintained in the flow-net log. To get the target event, the simulation traces from the parent event to its scope event. If this scope event has a scope pointer, the simulation traces to the scope event's scope event. If this scope event does not have a scope pointer, the simulation traces to its next event along the flow pointer in the flow until finding a scope pointer, and then trace to the scope event along the scope pointer. This process repeats until finding the target event. We set the time cost for tracing from an event to the successive event along the flow pointer in the same layer as a fixed value, $t_{e}$, and the time cost for tracing from an event to the child event in the lower layer along the scope pointer as a fixed value, $t_{c}$. The simulation selects 10 events in Layer 1 (the highest layer), and it calculates the time for finding the corresponding target events. The simulation result is shown in Fig. 12.

In the simulation for non-relation logging, a log file that records events in the four layers is generated. To get the target event, the simulation traces within the log. To be fair, we set the time cost for tracing from an event to the next event in the $\log$ as $t_{e}$ as well. The simulation selects the same 10 events as in the simulation for flow-net, and it calculates the time for finding the target events. The simulation result is shown in Fig. 12.

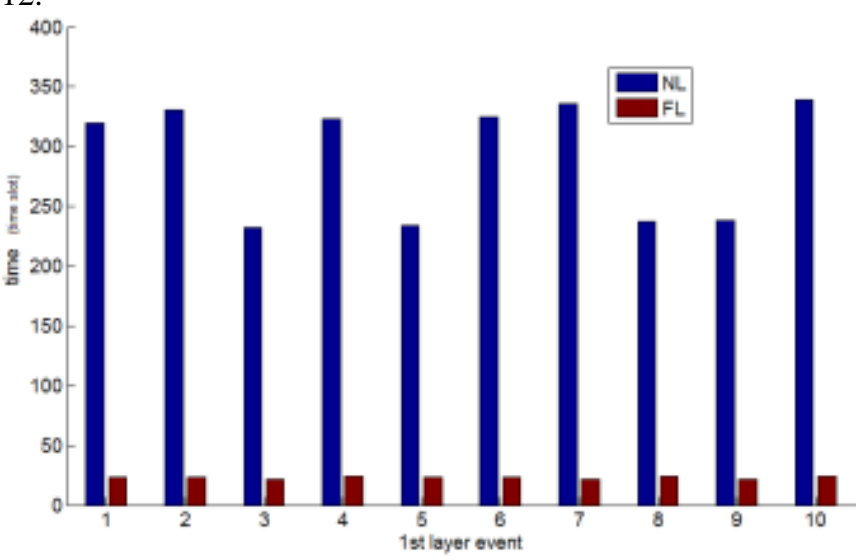

Fig. 12 Time cost of finding the 10 target events. The unit of the vertical axis is time slot. NL: No-relation logging; FL: flow-net logging.

Fig. 12 shows the time cost of finding the 10 target events that correspond to the 10 events at Layer 1 . In the simulation for non-relation logging, a scanning for the consecutive events in the log must be executed in order to locate the target events in the $\log$. In the simulation for flow-net, the benefit of the multi-resolution feature causes no time spent on scanning the large number of consecutive events in the log. With the multiresolution feature, we directly trace to and locate the scope events in the lower layer without costing time on scanning the events that are not related to the parent events. This is the reason why time cost for flow-net is much lower than that for non-relation logging in Fig. 12. This simulation result shows that multi-resolution flow-net provides better performance than non-relation logging.

This result also shows that the time costs for finding different events using flow-net are similar, but for non-relation logging are different. This implies that the time cost for finding an event using non-relation logging is affected by the location of the event in the log, but for finding an event using flow-net is not.

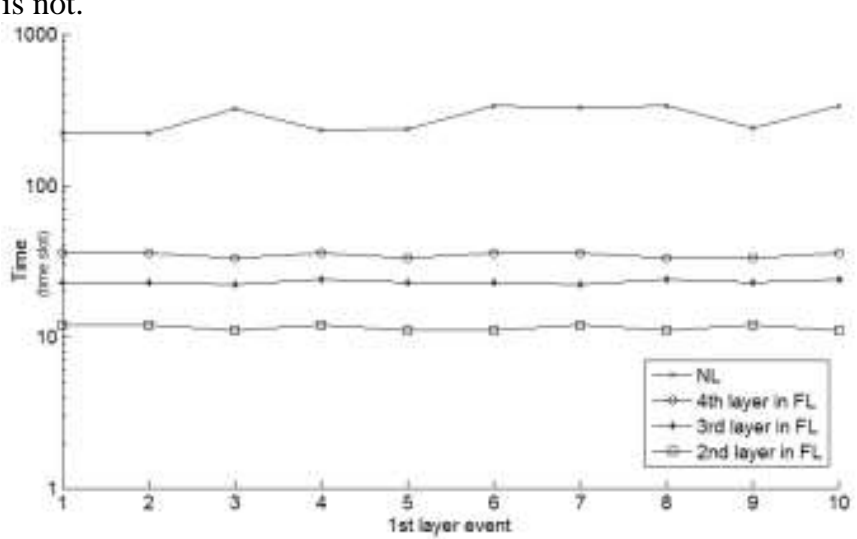

Fig. 13 Time cost of finding the events in different layers. The unit of the vertical axis is time slot. NL: No-relation logging; FL: flow-net logging.

Since the time cost for finding an event using flow-net is not affected by the location of the event in the log, we may want to know which factor influences the time cost. For this reason, we conduct another simulation that includes the same 10 events at layer 1 , and then, we calculate the time cost of finding the corresponding events in the second, third, and fourth layer.

Fig. 13 shows the time cost of finding the events in different lower layers using flow-net. The result implies that the layer number (e.g., 2nd, 3rd, and 4th) is a key factor that affects the time cost. This is because the depth of the layer determines how many times to trace along the scope pointers. Fig. 13 also shows that the time cost for using non-relation logging is always larger than the time cost for using flow-net. We may infer that the time costs for the two mechanisms are the same when the number of layers in flow-net approximately equals the number of events in the non-relation logging. However, this is a very extreme and rare situation since the number of events in the log is normally very large and the number of layers for events is normally small. This simulation result convinces us that multi-resolution flow-net provides a better performance than non-relation logging.

\section{Conclusion and Future Work}

In order to achieve different degrees of accountability for computer and network systems, we propose a multi-resolution flow-net. The mathematical definitions of flow-net are proposed. We propose different designs for multi-resolution flow-net, and we evaluate their performance and find the optimal design. TCP/IP networks and protocols are studied as the applications of multi-resolution flow-net. Our results show that the total time cost for tracing all of the related flow-net 
event records is proportional to the number of related records. The simulation result convinces us that multi-resolution flownet provides a better performance than non-relation logging.

Based on the relationship among events, flow-net can be beneficial for security related applications, such as authentication, access control, encryption, intrusion detection. However, accountable logging is a different feature /function from those security properties, and should be independently implemented and studied. In our future work, flow-net applications on security issues would be studied. We will study the security properties, along with many other security related topics, such as certificate authority, encryption, intrusion detection, and digital signature. We will also investigate how our proposed scheme applied on attack detection, such as distributed denial-of-service attack (DDoS), ICMP Attack, and IP spoofing attack. We will show how flow-net is able to maintain security properties in the future work

\section{Acknowledgement}

This work was supported in part by the US National Science Foundation (NSF) under grants CNS-0716211, CNS-0737325, CCF-0829827, and CNS-1059265.

\section{REFERENCES}

[1] Y. Xiao, "Flow-Net Methodology for Accountability in Wireless Networks," IEEE Network, Vol. 23, No. 5, Sept./Oct. 2009, pp. 30-37.

[2] D. J. Weitzner, H. Abelson, T. Berners-Lee, J. Feigenbaum, J. Hendler, and G. J. Sussman, "Information Accountability," Commun. ACM, vol. 51, pp. 82-87, 2008.

[3] J. Feigenbaum, A. Jaggard, R. Wright, and H. Xiao, "Systematizing 'Accountability' in Computer Science," Technical Report YALEU/DCS/TR-1452, 2012.

[4] B. Lampson, "Privacy and security: Usable security: how to get it," Commun. ACM, vol. 52, pp. 25-27, 2009.

[5] H. Chockler, and J. Y. Halpern, "Responsibility and Blame: A Structural-Model Approach," Journal of Artificial Intelligence Research, vol. 22, pp. 93-115, 2004.

[6] J. D. Ullman, "Principles of Database \& Knowledge-Base Systems," Computer Science Press, 1990.

[7] C. Lonvick, "The BSD syslog Protocol," RFC3164. Available: http://tools.ietf.org/html/rfc3164

[8] T. Park, S.B. Cho, and H.Y. Yeom, "An efficient logging scheme for recoverable distributed shared memory systems," Proc. of the 17th Int'l Conf. Distributed Computing Systems, May 1997.

[9] T. Park, N. Woo, H. Y. Yeom, "An Efficient Optimistic Message Logging Scheme for the Recoverable Mobile Computing Systems," IEEE Transactions on Mobile Computing, Vol. 1, 2001, pp. 265-277.

[10] Y. Xiao, K. Meng, and D. Takahashi, "Implementation and Evaluation of Accountability using Flow-net in Wireless Networks," Proceedings of the IEEE Military Communications Conference 2010 (IEEE MILCOM 2010).

[11] Y. Xiao, K. Meng, and D. Takahashi, "Accountability using Flow-net: Design, Implementation, and Performance Evaluation," (Wiley Journal of) Security and Communication Networks, Vol.5, NO. 1, pp. 29-49, Jan. 2012.

[12] R. Fielding, J. Gettys, J. Mogul, H. Frystyk, L. Masinter, P. Leach, T. Berners-Lee, "Hypertext Transfer Protocol -- HTTP/1.1," RFC 2616. Available: http://tools.ietf.org/html/rfc2616

[13] E. Rescorla, "HTTP Over TLS," RFC 2818. Available: http://tools.ietf.org/html/rfc2818

[14] Andrew S. Tanenbaum, "Computer Networks," 4th Edition, Published by Prentice Hall

[15] Inetdaemon, "TCP 3-Way Handshake." Available: http://www.inetdaemon.com/tutorials/internet/tcp/3way_handshake.shtml
[16] J. Postel, "User Datagram Protocol," RFC 768. Available: http://tools.ietf.org/html/rfc768

[17] Darpa Internet Program, "Internet Protocol”, RFC791. Available: http://en.wikipedia.org/wiki/IPv4

[18] Internet Protocol. http://en.wikipedia.org/wiki/Internet_Protocol

Available:

9] D. Comer, "Internetworking with TCP/IP Vol.1: Principles, Protocols, and Architecture", 4th ed. Prentice Hall, 2000.

[20] B. A. Forouzan, "Data Communications and Networking (Fourth edition)", McGraw-Hill, pp. 621-630, 2007.

[21] J. Erikson, "HACKING the art of exploitation (2nd ed.)," San Francisco: NoStarch Press, pp. 264

[22] B. Fu, and Y. Xiao, "An Implementation Scheme of Flow-Net and Its Applications on Detecting Attacks in Wireless Networks," Proc. of IEEE GLOBECOM 2010.

[23] A. Haeberlen, "A Case for the Accountable Cloud," ACM SIGOPS Operating Systems Review archive, Vol. 44, Issue 2, pp. 52-57, 2010.

[24] N. Kathiresshan, Z. Xiao, and Y. Xiao, "EagleEye: A Logging Framework for Accountable Distributed and Networked Systems," Proc. of INFOCOM WKSHPS, 2011.

[25] A.R. Yumerefendi and J.S. Chase, "The role of accountability in dependable distributed systems," Proc. of HotDep, 2005.

[26] A.R. Yumerefendi and J.S. Chase, "Trust but verify: accountability for network services," Proc. of 11th workshop on ACM SIGOPS, 2004.

[27] Department of Defense. Trusted Computer System Evaluation Criteria. Technical Report 5200.28-STD, Department of Defense, 1985.

[28] A.R. Yumerefendi and J.S. Chase, "Strong accountability for network storage," ACM Transactions on Storage, vol. 3, 2007.

[29] K. Argyraki, P. Maniatis, O. Irzak, S. Ashish, S. Shenker, and L. EPFL, "Loss and delay accountability for the Internet," IEEE International Conference on Network Protocols, pp. 194-205, 2007.

[30] B. Briscoe, A. Jacquet, T. Moncaster, and A. Smith, "Re-ECN: Adding Accountability for Causing Congestion to TCP/IP," IETF Internet-Draft, 2009.

[31] B. Briscoe, A. Jacquet, T. Moncaster, and A. Smith, "Re-ECN: The Motivation for Adding Accountability for Causing Congestion to TCP/IP”, IETF Internet-Draft, 2009.

[32] J. Mirkovic and P. Reiher, "Building accountability into the future Internet," Secure Network Protocols, 4th Workshop on, pp. 45-51, 2008.

[33] Y. Xiao, "Performance Analysis of Priority Schemes for IEEE 802.11 and IEEE 802.11e Wireless LANs," IEEE Transactions on Wireless Communications, Vol. 4, No. 4, pp. 1506-1515, Jul. 2005.

[34] Y. Xiao, "IEEE 802.11n: Enhancements for Higher Throughput in Wireless LANs," IEEE Wireless Communications, pp. 82-91, Dec. 2005.

[35] Y. Xiao, "IEEE 802.11e: A QoS Provisioning at the MAC layer," IEEE Wireless Communications, Vol. 11, No. 3, pp.72-79, Jun. 2004.

[36] S. Bechtold and A. Perrig, "Accountability in future internet architectures," Communications of the ACM, Vol. 57, Issue 9, pp. 2123, Sep. 2014

[37] J. Feigenbaum, A. Jaggard, and R. Wright, "Open vs. Closed Systems for Accountability," Proceedings of the 2014 Symposium and Bootcamp on the Science of Security (HotSoS '14), No. 4, 2014.

[38] C. Techapanupreeda, R. Chokngamwong, C. Thammarat, and S. Kungpisdan, "An accountability model for Internet transactions," International Conference on Information Networking (ICOIN), pp.127132, Jan. 2015.

[39] R. Gajanayake, T. Sahama, and R. Iannella, "Principles of Information Accountability: An eHealth Perspective," International Journal of EHealth and Medical Communications, Vol. 5, Issue 3, 2014.

[40] A. Datta, "Privacy through Accountability: A Computer Science Perspective," Distributed Computing and Internet Technology, Vol. 8337, pp. 43-49, 2014.

[41] S. Etalle and N. Zannone, "Understanding computer security," Frontiers in ICT, Vol. 1, 2014.

[42] Y. Xiao, "Accountability for Wireless LANs, Ad Hoc Networks, and Wireless Mesh Networks," IEEE Communication Magazine, Vol. 46, No. 4, Apr. 2008, pp. 116-126.

[43] L. Zeng, H. Chen, and Y. Xiao, "Accountable Administration and Implementation in Operating Systems," Proceeding of The IEEE Global Telecommunications Conference 2011 (IEEE GLOBECOM 2011). 
[44] Z. Xiao and Y. Xiao, "Accountable MapReduce in Cloud Computing,"in Proceedings of 2011 IEEE Conference on Computer Communications Workshops (INFOCOM WKSHPS), 2011, pp. 1082 - 1087.

[45] Z. Xiao and Y. Xiao, "Achieving Accountable MapReduce in Cloud Computing," (Elsevier) Future Generation Computer Systems, Vol. 30, No.1, Jan. 2014, pp. 1-13.

[46] J. Liu, Y. Xiao, and J. Gao, "Achieving Accountability in Smart Grids," IEEE Systems Journal, Vol. 8, No. 2, Jun. 2014, pp. 493-508.

[47] J. Liu and Y. Xiao, "An Accountable Neighborhood Area Network in Smart Grids," Proceedings of EMC 2012, pp 171-178.

[48] J. Liu, and Y. Xiao, "Temporal Accountability and Anonymity in Medical Sensor Networks," ACM/Springer Mobile Networks and Applications (MONET), Vol. 16, No. 6, pp. 695-712, Dec. 2011.

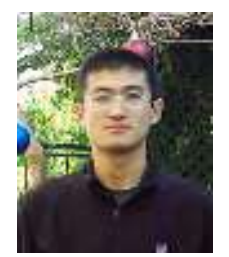

Bo Fu obtained his $\mathrm{Ph} . \mathrm{D}$. degree in computer science at the University of Alabama in 2013. He obtained the bachelor degree in Computer Science from Harbin Institute of Technology, China, in 2007. He currently works at Cisco, USA. His research interests are in security and accountability of computer networks.

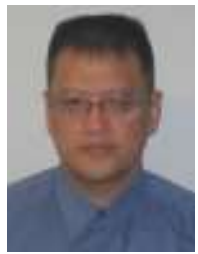

Yang Xiao currently is a Professor of Department of Computer Science at the University of Alabama, Tuscaloosa, AL, USA. His current research interests include communications/networks and computer/network security. He has published over 200 SCI-indexed journal papers and over 200 EI indexed refereed conference papers and book chapters related to these research areas. His research has been supported by the U.S. NSF, U.S. Army Research, GENI, Fleet Industrial Supply Center-San Diego, FIATECH, and The University of Alabama's Research Grants Committee. Dr. Xiao was a Voting Member of IEEE 802.11 Working Group from 2001 to 2004, involving IEEE 802.11 (WIFI) standardization work. He is a Fellow of IET (FIET). He served/serves as a Panelist for the U.S. National Science Foundation (NSF), The Global Environment for Network Innovations (GENI), Canada Foundation for Innovation's Telecommunications expert committee, and the American Institute of Biological Sciences, as well as a Referee/Reviewer for many national and international funding agencies. He currently serves as Editor-in-Chief for International Journal of Security and Networks, International Journal of Sensor Networks, and Journal of Communications. He had (s) been an Editorial Board or Associate Editor for 15 international journals, including IEEE Transactions on Systems, Man, and Cybernetics: Systems, during 2014 to 2015, IEEE Transactions on Vehicular Technology, during 2007 to 2009, and IEEE Communications Survey and Tutorials, during 2007 to 2014. He served (s) as a Guest Editor for over 20 times for different international journals, including IEEE Network, IEEE Wireless Communications, and ACM/Springer Mobile Networks and Applications (MONET). Dr. Xiao has delivered over 30 keynote speeches at international conferences around the world and gave more than 60 invited talks at different international institutes. 